\title{
Perfil Epidemiológico de Usuários do Serviço de Farmácia Comunitária no Bairro de Santíssimo, Zona Oeste do Rio de Janeiro
}

\section{Epidemiological Profile of Users of the Community Pharmacy Service in the District the Santíssimo, West Zone of Rio de Janeiro}

\author{
Márcio Vinícius de Abreu Verli ${ }^{1 *}$, Luis Carlos Oliveira Gonçalves $1^{1}$, Raphael Benassi $2^{2}$, \\ Marcelle Karyelle Montalvão Gomes $3^{1}$, Aníbal Monteiro de Magalhães Neto $4^{1}$
}

\begin{abstract}
RESUMO
As doenças cardiovasculares causam aproximadamente 15 milhões de mortes por ano no mundo. Inquéritos de saúde apresentam soluções para se construir indicadores associados à saúde e ainda obter informações de fatores de risco e determinantes sociais do processo saúde/doença. Índices antropométricos como o índice de massa corporal são utilizados como preditores de risco das principais doenças cardiovasculares. O objetivo do nosso estudo foi o de traçar o perfil epidemiológico de uma determinada população, indicando como objetivos específicos as doenças prevalentes, índices antropométricos, além dos valores de pressão arterial e glicemia capilar da amostra em questão. Participaram deste estudo 82 indivíduos, sendo 31 do gênero masculino e 51 do gênero feminino, com idades entre 21 e 78 anos. Quando analisamos o IMC verificamos que $20 \%$ do total da amostra encontra-se no grupo de indivíduos até 25 (normal), $40 \%$ no grupo de 25,1 até 30 (sobrepeso), 28\% no grupo de 30,1 até 35 (obesos) e 12\% no grupo de $>35$ (obesos mórbidos). Ao analisarmos os valores da pressão arterial sistólica observamos que os dados são preocupantes, por apresentarem suas médias, para todos os grupos etários, superiores a 120 mmhg (média de 137,1 mmhg). Nosso estudo demonstra a necessidade de inserções políticas visando a promoção de saúde e adequação do serviço público a realidade atual da população em questão.
\end{abstract}

Palavras-chave: Perfil epidemiológico; Pressão arterial; Saúde pública.

\section{ABSTRACT}

Cardiovascular diseases cause approximately 15 million deaths worldwide each year. Health surveys present solutions to build indicators associated with health and also to obtain information on risk factors and social determinants of the health/disease process. Anthropometric indices such as body mass index are used as risk predictors of the main cardiovascular diseases. The objective of our study was to trace the epidemiological profile of a given population, indicating as specific objectives the prevalent diseases, anthropometric indices, in addition to the blood pressure and capillary glucose values of the sample in question. A total of 82 individuals participated in this study, 31 males and 51 females, aged between 21 and 78 years. When we analyzed the BMI, we found that $20 \%$ of the total sample is in the group of individuals up to 25 (normal), $40 \%$ in the group from 25.1 to 30 (overweight), $28 \%$ in the group from 30.1 to 35 (obese) and $12 \%$ in the $>35$ group (morbidly obese). When analyzing the values of systolic blood pressure, we observed that the data are worrying, as they present their averages, for all age groups, above $120 \mathrm{mmhg}$ (mean of $137.1 \mathrm{mmhg}$ ). Our study demonstrates the need for political insertions aimed at promoting health and adapting the public service to the current reality of the population in question.

Keywords: Epidemiological profile; Blood pressure; Public health.

\footnotetext{
${ }^{1}$ Universidade Federal de Mato Grosso - UFMT.

${ }^{2}$ Benassi pro training

*E-mail: marcioaverli@gmail.com
} 


\section{INTRODUÇÃO}

As doenças cardiovasculares (DCV) causam aproximadamente 15 milhões de mortes por ano no mundo, correspondendo a 30\% do total de óbitos (LAURENTI e BUCHALLA, 2001). Estas podem ser associadas às dislipidemias (LESSA et al., 1997), hipertensão arterial (PIERIN et al., 2001), valvopatias (KRUCZAN et al., 2008) e a fatores de risco modificáveis como tabagismo e sedentarismo (RODRIGUES, CHEIK e MAYER, 2008).

A descontinuidade do tratamento é um problema observado na maioria das doenças crônicas. Vários determinantes atuam na problemática da adesão (PIERIN et al., 2001). Inquéritos de saúde apresentam soluções práticas para se construir indicadores associados à saúde, e ainda obter informações de fatores de risco e determinantes sociais do processo saúde/doença (PEREIRA, BARRETO e PASSOS, 2008).

Além dos inquéritos, outras ferramentas são amplamente utilizadas pela sua associação com a prevalência de doenças. Índices antropométricos como o índice de massa corporal (IMC) são utilizados como preditores de risco das principais doenças cardiovasculares (GUS et al., 1998).

Determinar o perfil epidemiológico de diferentes populações auxilia a compreensão da prevalência de doenças em diferentes regiões geográficas e níveis sociais (DANILOW et al., 2007).

Estudos de perfil na área de saúde facilitam processos de mudança no planejamento em saúde e melhoram a assistência a diferentes regiões de acordo com suas necessidades específicas (GARCIA e SAINTRAIN, 2009).

O objetivo geral do nosso estudo foi o de traçar o perfil epidemiológico de uma determinada população, indicando como objetivos específicos as doenças prevalentes, índices antropométricos, além dos valores de pressão arterial e glicemia capilar da amostra em questão.

\section{METODOLOGIA}

Participaram deste estudo 82 (oitenta e dois) indivíduos, sendo 31 (trinta e um) do gênero masculino e 51 (cinquenta e um) do gênero feminino, com idades entre 21 (vinte e um) e 78 (setenta e oito) anos (DP 12,52; EP 1,38). 
A amostra foi constituída por voluntários que participaram do evento dia da saúde, que ocorreu no mês de junho/2012, com atendimento à população usuária do serviço de farmácia comunitária no bairro de santíssimo, zona oeste do Rio de Janeiro.

Tabela 1. Características da amostra.

\begin{tabular}{lcccccccc} 
& IDADE & PESO & ESTATURA & IMC & P.A.S. & P.A.D. & GLICEMIA & FPM \\
MÉDIA & 54,47 & 76,95 & 1,62 & 29,14 & 137,1 & 90,85 & 129,3 & 26,95 \\
MEDIANA & 57,0 & 75,35 & 1,61 & 28,55 & 130,0 & 90,0 & 117,0 & 26,0 \\
D.P. & 12,5 & 16,78 & 0,09 & 5,47 & 21,05 & 14,16 & 48,4 & 7,53 \\
E.P. & 1,38 & 1,85 & 0,01 & 0,60 & 2,33 & 1,56 & 5,34 & 0,83 \\
\hline
\end{tabular}

Idade em anos, peso em Kilogramas, estatura em metros, pressão em milímetros de mercúrio, glicemia em miligrama por decilítro e força de preensão manual em Kilograma/força.

Os valores de glicemia não foram discutidos pelo fato do não cumprimento do jejum de 8 (oito) horas pela amostra em questão, dificultando a análise dos resultados obtidos.

O peso foi obtido através de balança digital da marca GTECH® com precisão de $0,1 \mathrm{Kg}$. A estatura por estadiômetro Personal da marca SANNY® com graduações em 2 milímetros. O índice de massa corporal (IMC) foi calculado por (PESO/ESTATURA ${ }^{2}$ ). A pressão arterial foi obtida a partir de esfigmomanómetro analógico aneróide da marca PREMIUM®. A glicemia por medidor de glicemia capilar GTECH® e a Força de Preensão Manual (FPM) foi obtida a partir de dinamômetro de preensão manual JAMAR®.

Os indivíduos foram divididos em grupos por idade, sendo GRUPO 1 (20 - 25 anos); GRUPO 2 (26 - 30 anos); GRUPO 3 (31 - 35 anos); GRUPO 4 (36 - 40 anos); GRUPO 5 (41 45 anos); GRUPO 6 (46 - 50 anos) e GRUPO 7 (>50 anos).

\section{RESULTADOS E DISCUSSÃO}

Tabela 2. Valores de IMC, PAS e FPM estratificado por grupos e gênero.

\begin{tabular}{|l|l|l|l|l|l|l|l|l|l|l|l|}
\hline IMC & Total & M & F & PAS & Total & M & F & FPM & Total & M & F \\
\hline Até 25 & $16(20)$ & 8 & 8 & Até 120 & $28(34)$ & 8 & 20 & $<\mathbf{2 0}$ & $12(15)$ & 2 & 10 \\
\hline $\mathbf{2 5 , 1 - 3 0}$ & $33(40)$ & 13 & 20 & $\mathbf{1 2 1 - 1 3 0}$ & $16(20)$ & 6 & 8 & $\mathbf{2 0 - 3 0}$ & $47(57)$ & 10 & 37 \\
\hline $\mathbf{3 0 , 1 - 3 5}$ & $23(28)$ & 9 & 14 & $\mathbf{1 3 1 - 1 4 0}$ & $13(16)$ & 8 & 5 & $\mathbf{3 0 , 1 - 4 0}$ & $18(22)$ & 14 & 4 \\
\hline $\mathbf{3 5}$ & $10(12)$ & 1 & 9 & $>\mathbf{1 4 0}$ & $25(30)$ & 9 & 16 & $\mathbf{> 4 0}$ & $5(6)$ & 5 & 0 \\
\hline
\end{tabular}

Quantidade de indivíduos por grupo de índice de massa corporal (\% do total); Pressão arterial sistólica (\% do total) e Força de Preensão Manual (\% do total), masculino(M); feminino(F).

Quando analisamos o IMC verificamos que $20 \%$ do total da amostra encontra-se no grupo de indivíduos até 25 (normal), 40\% no grupo de 25,1 até 30 (sobrepeso), 28\% no grupo de 30,1 até 35 (obesos) e 12\% no grupo de >35 (obesos mórbidos). 
Gráfico 1. Média e erro padrão da Força de Preensão Manual por grupo etário.

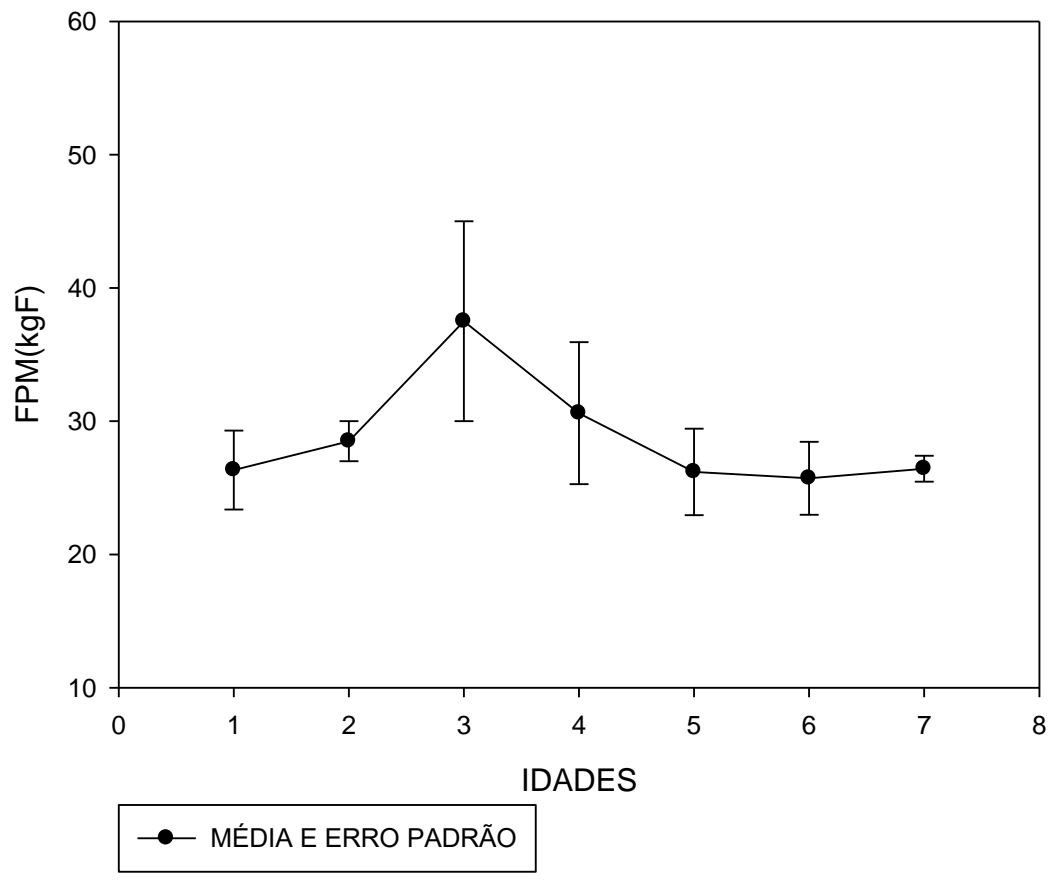

Foi observado um acréscimo de força, tendo seu pico na faixa de 31 a 35 anos de idade, corroborando assim com achados anteriores de estudos feitos com indivíduos australianos (MASSY-WESTROPP, et al., 2004), e com amostra brasileira obtida a partir dos dados de estudo populacional (CAPORRINO, et al., 1998). Esse comportamento da curva de força de preensão manual pela idade já havia sido mostrado anteriormente pelo nosso grupo (GONÇALVES, et al., 2012), onde para atletas as médias alcançaram valores superiores, mas com um mesmo comportamento para a linha do tempo. Estes valores podem servir de base para análise de diferentes amostras dentro da população brasileira, facilitando esta observação por profissionais de saúde e pesquisadores em saúde pública.

Gráfico 2. Média e erro padrão da pressão arterial sistólica por grupo etário. 


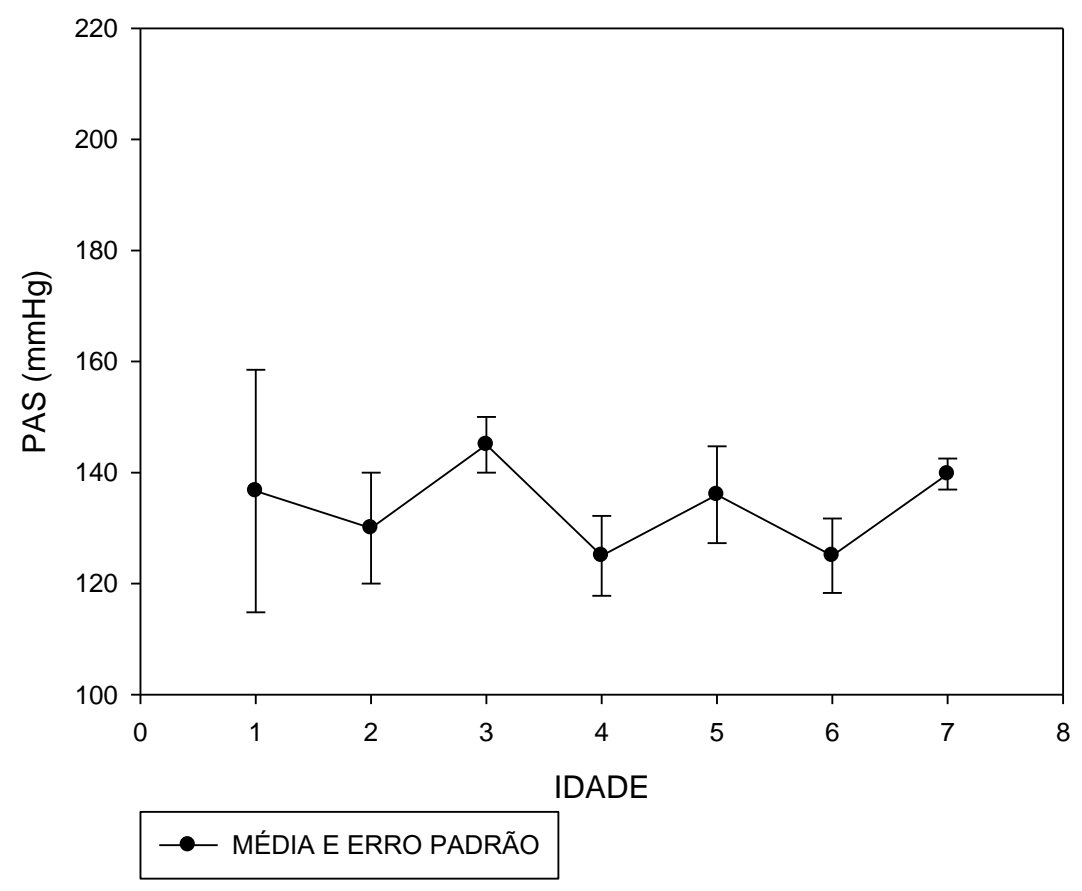

Ao analisarmos os valores da pressão arterial sistólica observamos que os dados são preocupantes, por apresentarem suas médias, para todos os grupos etários, superiores a $120 \mathrm{mmhg}$ (média de 137,1 mmhg). Isso indica um elevado índice de hipertensão arterial na amostra obtida, com pouca variação por grupo de idade. 
Gráfico 3. Média e erro padrão do índice de massa corporal por grupo etário.

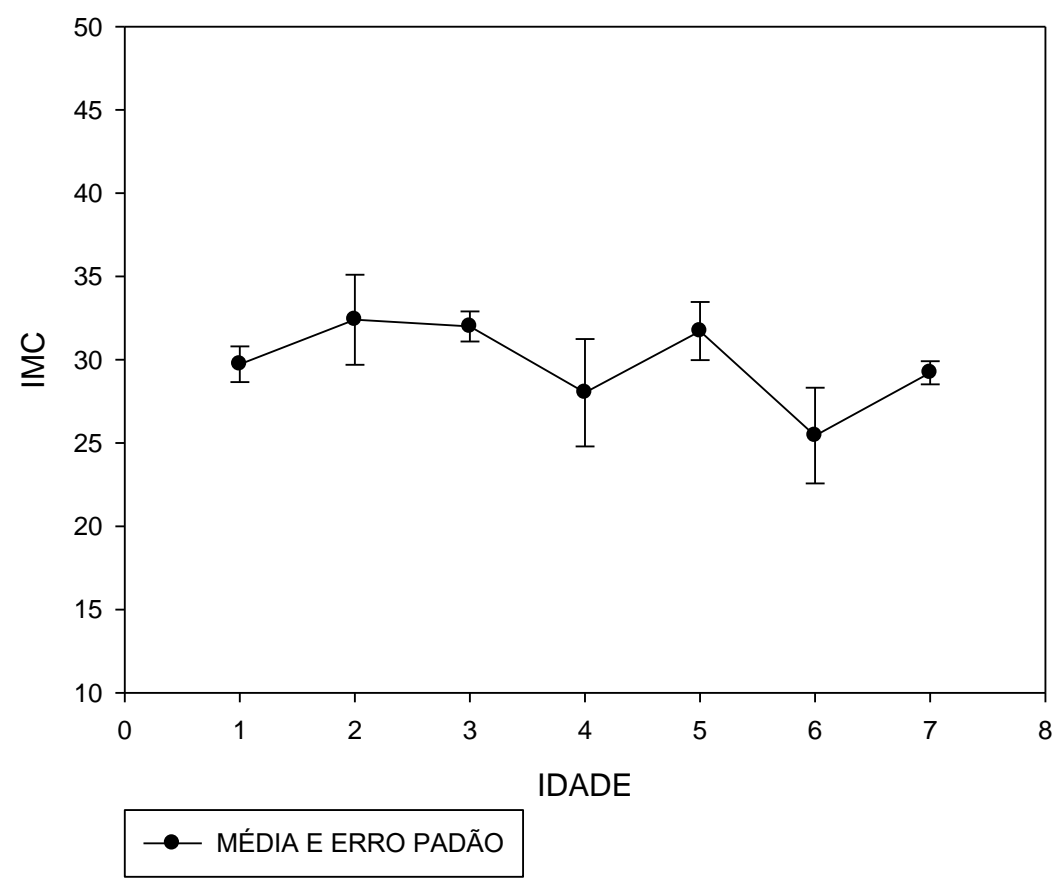

Um outro dado que requer atenção é o índice de massa corporal da nossa amostra, pois este apresentou valores de médias superiores ao ideal que é de até 25 (sobrepeso), chegando em alguns grupos alcançar valores superiores a 30, que é considerado de obesidade (WHO, 2000). Esse dado se mostra relevante, pois de acordo com Anjos e colaboradores (1998) na adolescência há uma elevação dos valores de IMC devido ao desenvolvimento corporal, porém estes valores na fase adulta não devem ultrapassar 25 , sendo esta elevação um fator de risco cardiovascular.

\section{CONSIDERAÇÕES FINAIS}

Estudos de perfil epidemiológico apresentam resultados que podem ser apreciados e analisados por profissionais de saúde, orgão públicos e pesquisadores para promover políticas públicas, direcionando-as de forma adequada a cada população.

Nosso estudo demonstra a necessidade de inserções políticas visando a promoção de saúde e adequação do serviço público a realidade atual da população em questão.

Programas de atividade física podem ser inseridos em cada bairro, descentralisando estes programas dos grandes centros esportivos da prefeitura, atingindo uma maior quantidade de indivíduos. 


\section{REFERÊNCIAS}

ANJOS, L. A.; VEIGA, G. V.; CASTRO, I. R. R. Distribuição dos valores do índice de massa corporal da população brasileira até 25 anos. Revista Panamericana de Saúde Pública. v. 3, n.3, p. 164-173, 1998.

CAPORRINO, F. A.; FALOPPA, F.; SANTOS, J. B. G. dos; RÉSSIO, C.; SOARES, F. H. de C.; NAKACHIMA, L. R.; SEGRE, N. G. Estudo populacional da força de preensão palmar com dinamômetro JAMAR. Revista Brasileira de Ortopedia. v. 33, n. 2. 1998.

DANILOW, M. Z.; MOREIRA, A. C. S.; VILELA, C. G.; BARRA, B. B.; NOVAES, M. R. C. G.; OLIVEIRA, M. P. F. Perfil epidemiológico, sociodemográfico e psicossocial de idosos institucionalizados do Distrito Federal. Com. Ciências da Saúde. v. 18, n.3, p. 9-16, 2007.

GARCIA, S. S. \& SAINTRAIN, M. V. L. Perfil epidemiológico de uma população idosa atendida pelo programa de saúde da família. Revista de Enfermagem da UERJ. v. 17, n. 1, p. 18-23, 2009.

GONÇALVES, L. C. O.; BENASSI, R.; OLIVEIRA, A. L. B. Valores de referência de força de preensão manual para homens praticantes de judô. Revista Brasileira de Prescrição e Fisiologia do Exercício. v.6, n.32, p. 128-132, 2012.

GUS, M.; MOREIRA, L. B.; PIMENTEL, M.; GLEISENER, A. L. M.; MORAES, R. S.; FUCHS, F. D. Associação entre diferentes indicadores de obesidade e prevalência de hipertensão arterial. Arquivos Brasileiros de Cardiologia. v. 70, n. 2, p. 111-114, 1998.

KRUCZAN, D. D.; SOUZA E SILVA, N. A.; PEREIRA, B. B.; ROMÃO, V. A.; FILHO, W. B. C.; MORALES, F. E. C. Doença Arterial Coronariana em Pacientes com Valvopatia Reumática e Não-Reumática Acompanhados em Hospital Público do Rio de Janeiro. Arquivos Brasileiros de Cardiologia. v. 90, n. 3, p. 217-223, 2008.

LAURENTI, R. \& BUCHALLA, C. M. Os mitos a respeito das doenças cardiovasculares. Arquivos Brasileiros de Cardiologia. v. 76, n. 2, 2001.

LESSA, I.; CONCEIÇÃO, J. L.; SOUZA, M. L.; OLIVEIRA, V.; CARNEIRO, J.; MELO, J.; PINHEIRO, J.; MEIRELES, F.; NETTO, J.; REIS, F.; GOUVÊA, R.; COUTO, M.; SOUZA, S. \& OLIVEIRA, M. R. Prevalência de dislipidemias em adultos da demanda laboratorial de Salvador, Brasil. Arquivos Brasileiros de Cardiologia. v. 69, n. 6, p. 395-400, 1997. 
MASSY-WESTROPP, N.; RANKIN, W.; AHEN, M.; KRISNHAN, J.; HEAN, T. Measuring Grip Strength in Normal Adults: Reference Ranges and a Comparison of Electronic and Hydraulic Instruments. The Journal of Hand Surgery. v. 29, n. 3, 2004.

PEREIRA, J. C.; BARRETO, S. M. \& PASSOS, V. M. O Perfil de Saúde Cardiovascular dos Idosos Brasileiros Precisa Melhorar: Estudo de Base Populacional. Arquivos Brasileiros de Cardiologia, v. 91, n. 1, p. 1-10, 2008.

PIERIN, A. M. G.; MION Jr, D.; FUKUSHIMA, J. T.; PINTO, A. R.; KAMINAGA, M. M. O perfil de um grupo de pessoas hipertensas de acordo com o conhecimento e a gravidade da doença. Revista da Escola de Enfermagem - USP. v. 35, n. 1, p. 11-18, 2001.

RODRIGUES, E. S. R.; CHEIK, N. C.; MAYER, A. F. Nível de atividade física e tabagismo em universitários. Revista de Saúde Pública. v. 42, n. 4, p. 672-678, 2008.

WORLD HEALTH ORGANIZATION (WHO). Obesity: preventing and managing the global epidemic. Report of a WHO Consultation. WHO Technical Report Series 894. Geneva; 2000. 